\title{
Using LoRa Networks \\ in Automatic Band Dendrometer
}

\author{
Yuri N. Bezborodov and Ilya N. Rubtsov* \\ Siberian Federal University \\ Krasnoyarsk, Russian Federation
}

Received 19.07.2021, received in revised form 30.07.2021, accepted 05.08.2021

\begin{abstract}
This article describes the experience of using wireless LoRa data transfer in an automatic band dendrometer, developed by the author early [1].

Previously was developed an automatic band dendrometer with web data storage, we provided information about the process of creating the device and the result of its testing.

As the result of the tests, the necessary improvements were formulated, one of which was the need to use a network of several sensors located in one area for measurements and transmit their measurements wirelessly to a central data logger for processing and transmission to the web server.

Thus, it became necessary to select and implement an energy-saving data exchange protocol with a sufficient range and reliable performance in a taiga.
\end{abstract}

Keywords: dendrometer, LoRa, wireless sensor.

Citation: Bezborodov Yu.N., Rubtsov I. N. Using LoRa networks in automatic band dendrometer, J. Sib. Fed. Univ. Eng. \& Technol., 2021, 14(5), 600-605. DOI: 10.17516/1999-494X-0337

\section{Использование сетей LoRa}

\section{в автоматическом цифровом дендрометре}

\author{
Ю.Н. Безбородов, И.Н. Рубцов \\ Сибирский федеральньй университет \\ Российская Федеращчи, Красноярск
}

Аннотация. В данной статье описывается опыт использования сетей передачи данных стандарта LoRa в автоматическом кольцевом дендрометре, разработанном собственными силами и описанном paнee [1].

(C) Siberian Federal University. All rights reserved

This work is licensed under a Creative Commons Attribution-Non Commercial 4.0 International License (CC BY-NC 4.0).

* Corresponding author E-mail address: 3507777@gmail.com

ORCID: 0000-0001-5111-9050 (Rubtsov) 
Ранее нами был разработан и представлен автоматический кольцевой дендрометр с отправкой данных на сервер в интернете, был описан процесс создания прибора и результат его испытаний. В результате испытаний были сформулированы необходимые доработки, одной из которых была необходимость использовать для измерений сеть из нескольких датчиков, расположенных на одном участке, и передачу их измерений беспроводным способом на центральный дата логгер для обработки и передачи на сервер.

Таким образом, появилась необходимость выбрать и внедрить энергосберегающий протокол обмена данными, обладающий достаточным радиусом действия и надежностью работы в условиях тайги.

Ключевые слова: дендрометp, LoRa, беспроводной датчик.

Цитирование: Безбородов, Ю.Н. Использование сетей LoRa в автоматическом цифровом дендрометре / Ю. Н. Безбородов, И.Н. Рубцов // Журн. Сиб. федер. ун-та. Техника и технологии, 2021, 14(5). С. 600-605. DOI: 10.17516/1999-494X-0337

Данная статья является продолжением исследования по разработке программноаппаратного комплекса для дендрометрии, состоящего из автоматического кольцевого дендрометра или сети дендрометров и центрального узла - дата логгера, обрабатывающего и передающего информацию на сервер в интернете.

Ранее было разработано несколько опытных образцов как проводных, так и беспроводных дендрометров [1], проведены их полевые испытания [2], в результате которых были получены положительные результаты их работы и сформулированы пути модернизации и улучшения характеристик приборов.

Одной из слабых сторон предыдущей версии беспроводного дендрометра было использование в нем для передачи данных сети ZigBee 2.4 ГГц, которая хоть и обеспечивала функционирование, но давала ограниченный радиус действия (не более 50 м), а в случае с применением сети датчиков выход из строя одного из них мог нарушить стабильную работы всей системы.

Было решено найти альтернативу, позволяющую при низком энергопотреблении увеличить радиус действия и сохранить независимость каждого отдельного датчика в передаче данных на дата логгер.

Сейчас наблюдается интенсивное развитие так называемого Интернета вещей, связанное с быстрым развитием энергосберегающих технологий передачи данных, позволяющих устройствам с батарейным питанием продолжительное время оставаться автономными, не прекращая передачу данных. Но эти технологии имеют разные технические принципы работы и свои плюсы и минусы. Был проведен сравнительный анализ сетей беспроводной передачи данных с увеличенным диапазоном действия.

Основные используемые сейчас энергосберегающие сети передачи данных с увеличенным радиусом действия - это LoRa, NB-IoT и Wi-Fi HaLow (802.11ah).

Среди представленных сетей NB-IoT работает в диапазоне частот LTE (таких как 25302540, 2650-2660, 847-854.5 МГц) и имеет виды модуляции: OFDM, BPSK, QPSK, QAM. Данный диапазон частот является лицензируемым в России, и работа устройств требует наличие базовой станции, что не подходит для использования в удаленной местности. Максимальная чувствительность приемника составляет -141 дБм, что является лучшим показателем из трех представленных технологий. Максимальная мощность передатчика во всех трех случаях -500 мВт. Дальность связи ограничена покрытием сети и количеством базовых станций.

$$
-601-
$$


Cтандарт LoRa использует нелицензируемый диапазон частот, который может изменяться в зависимости от местных законов страны применения. В России для него выделен диапазон частот 864-865 и 868.7-869.2 МГц. LoRa имеет собственную модуляцию, основанную на технологии расширения спектра и вариации линейной частотной модуляции с интегрированной прямой коррекцией ошибок. Чувствительность приемника составляет от -108 до -136 дБм. Дальность связи - до 10 км.

Стандарт WiFi HaLow (802.11ah) также использует нелицензируемый в России диапазон частот. Оценочная чувствительность приемника составляет от -120 до -130 дБм. Дальность связи - до 1 км.

В итоге к особенностям первой технологии можно отнести высокую скорость связи, высокое качество передачи данных, но большую стоимость оборудования, необходимость лицензирования частот и наличие SIM-карты.

Особенности второй технологии - низкая стоимость оборудования, большой радиус действия, низкое энергопотребление, закрытый стандарт связи.

Oсобенности WiFi HaLow - меньший (чем у предыдущих двух) радиус действия, возможность интеграции с имеющимися сетями WiFi.

Таким образом, проанализировав преимущества и недостатки всех трех стандартов, учитывая необходимость использования в труднодоступных регионах, приняли решение применять стандарт LoRa в автоматическом дендрометре.

B качестве аппаратной реализации стандарта LoRa для использования в данном проекте был задействован трансивер SX1278 компании Semtech. Данный трансивер работает на частоте 433МГц, обладает скоростью передачи данных до 300 Кбит/с и низким энергопотреблением, около 9,9 mА в режиме передачи.

Для упрощения разработки экспериментального образца был использован готовый модуль на основе данного трансивера, в котором все радиочастотные цепи и компоненты, необходимые для корректной работы, были уже размещены на предприятии-изготовителе. Внешний вид модуля представлен на рис. 1.

С использованием данного модуля была разработана электрическая схема, проект печатной платы и модифицированная прошивка микроконтроллера устройства. Модуль не занимает значительного пространства, поэтому печатная плата разместилась в габаритах прежнего кор-

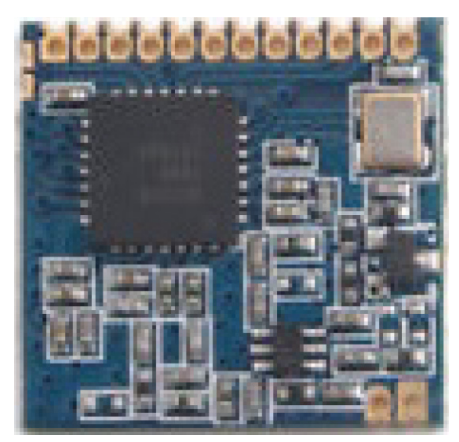

Рис. 1. Внешний вид LoRa-модуля

Fig. 1. LoRa module 
пуса дендрометра. Была проведена защита платы от попадания влаги путем покрытия собранной печатной платы защитным составом. Также блок с электронными компонентами в корпусе дендрометра защищен от попадания воды.

В качестве аккумулятора использовался Li-ion аккумулятор емкостью $2500 \mathrm{mAh}$, обеспечивающий устройство питанием. Внешний вид собранного дендрометра представлен на рис. 2.

Для тестирования стабильности работы два дендрометра были размещены на участке на удалении около 1 км друг от друга и центрального дата логгера. Тестирование автономной работы производилось на протяжении 1 месяца с периодическим промежуточным контролем работы. Интервал отправки данных с дендрометра на центральный дата логгер составлял 30 мин, измерения изменения диаметра ствола дерева, температуры окружающей среды и влажности производились один раз в 5 мин. Данные, полученные с прибора, приведены на графике рис. 3.

Для экспериментальной оценки качества связи была проведена серия измерений на разном расстоянии от центрального дата логгера.

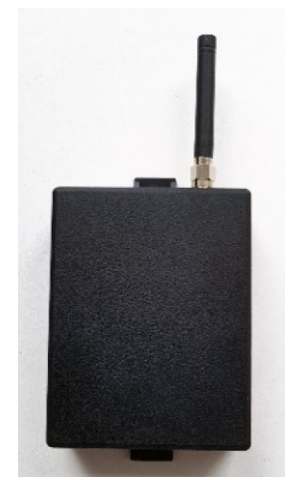

Рис. 2. Внешний вид дендрометра с LoRa-трансивером

Fig. 2. Dendrometer with LoRa transceiver
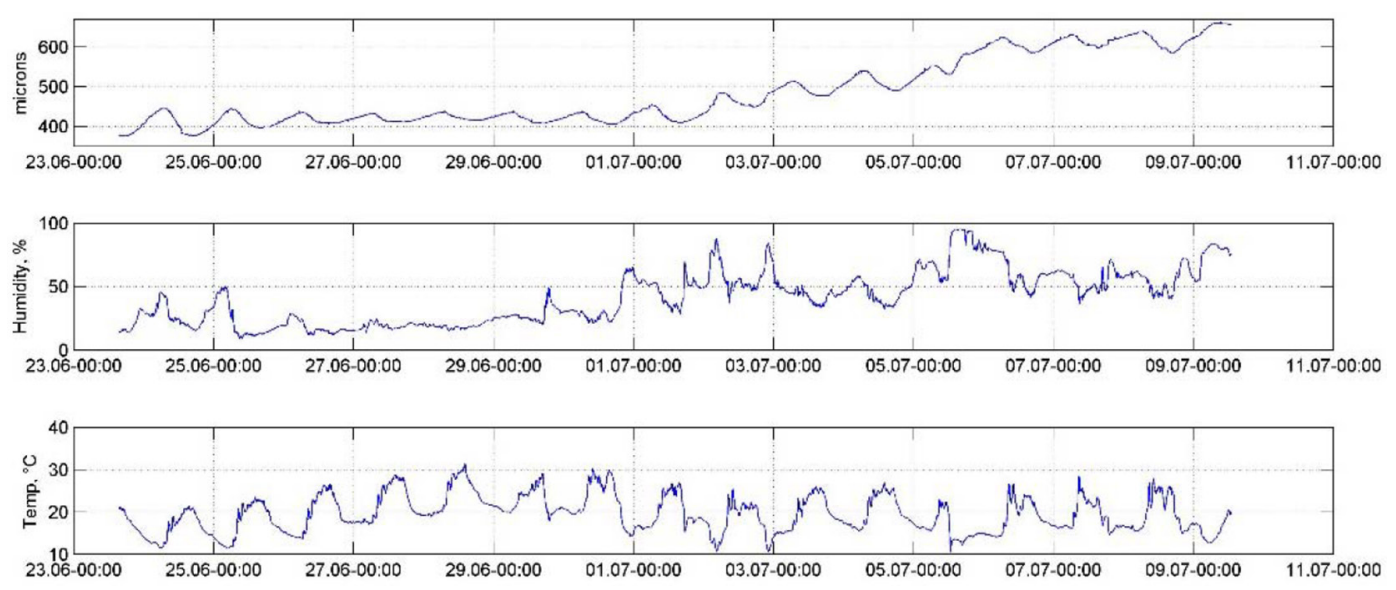

Рис. 3. Данные с дендрометра во время испытаний

Fig. 3. Dendrometer data during testing

$$
-603-
$$


Таблица 1. Зависимость качества связи от расстояния

Table 1. Dependence of quality due to the distance

\begin{tabular}{|c|c|c|c|}
\hline № изм. & Расстояние, м & RSSI, dBm & PER, \% \\
\hline 1 & 1105 & -112 & 0 \\
\hline 2 & 1310 & -115 & 1 \\
\hline 3 & 1620 & -110 & 1,1 \\
\hline 4 & 2252 & -121 & 0,3 \\
\hline 5 & 2523 & -119 & 0,5 \\
\hline 6 & 2850 & -129 & 0,6 \\
\hline 7 & 2975 & -115 & 2,15 \\
\hline 8 & 3351 & -127 & 3,5 \\
\hline 9 & 3520 & -130 & 0 \\
\hline
\end{tabular}

Уровень качества соединения оценивался по уровню сигнала RSSI (Received Signal Strength Indicator). Также использовался показатель процентного соотношения числа недоставленных пакетов к успешно доставленным PER (Packet Error Rate). В каждой точке удаленности производилась передача около 100 пакетов данных. Передатчик и приемник находились в 1,5 м от земли. Перепад высот при изменении удаления передатчика от приемника составлял не более 3 м. Данные изменений уровня сигнала, PER, представлены в табл. 1. Значения с PER более 4 (при расстоянии более 4000 м) отбрасывали и в таблицу не вносили.

Из анализа полученных данных можно сделать вывод, что с использованием данных антенн при таком расположении дендрометра и дата логгера уверенный радиообмен сохраняется на расстоянии около 3,5 км.

Выводы. После получения всех результатов был проведен анализ по временным меткам на предмет стабильности передачи данных, в результате которого было определено, что все измерения успешно передавались через радиоканал на дата логгер. Это позволяет говорить об успешном использовании сети передачи данных LoRa в условиях автономной работы в тайге.

Низкое энергопотребление в комбинации с большим радиусом действия делает низкую себестоимость сети LoRa удачным решением для тех задач, где требуется передавать небольшой объем данных, не критичный к скорости передачи на удаленные расстояния.

\section{Список литературы / References}

[1] Рубцов И.Н. Автоматический измеритель приращения диаметра ствола дерева на основе абсолютного магнитного цифрового энкодера. Контроль. Диагностика. 2016, 4, 6468. [Rubtsov I.N. The automatic tree trunk diameter increment meter based on the absolute magnetic digital encoder Testing. Diagnostics. 2016, 4, 64-68 (in Russian)]

[2] Рубцов И.Н. Результаты полевых испытаний разработанного автоматического кольцевого дендрометра с цифровым измерительным элементом. Известия Тульского государственного университета. Технические науки. 2016, 3, 90-96. [Rubtsov I.N. Automatic tree trunk diameter increment meter based on absolute magnetic digital encoder. Izvestiya Tula State University (Izvestiya TulGU) 2016, 3, 90-96 (in Russian)]

$$
-604-
$$


[3] Князев Н.С., Чечеткин В.А., Летавин Д.А. Сравнительный анализ стандартов беспроводных сетей LPWAN. Системы синхронизации, формирования и обработки сигналов. 2017, 2, 109-112 [Knyazev N. S., Chechetkin V. A., Letavin D. A. Comparative analysis of standards for wireless networks LPWAN. Systems of synchronization, formation and processing of signals. 2017, 2, 109-112 (in Russian)] 